\title{
Regulation of apoptosis by p53-inducible transmembrane protein containing sushi domain
}

\author{
HONGYAN CUI, HIROKI KAMINO, YASUYUKI NAKAMURA, NORIAKI KITAMURA, \\ TAKAFUMI MIYAMOTO, DAISUKE SHINOGI, OLGA GODA, \\ HIROFUMI ARAKARA and MANABU FUTAMURA
}

Cancer Medicine and Biophysics Division, National Cancer Center Research Institute, 5-1-1 Tsukiji, Chuo-ku, Tokyo 104-0045, Japan

Received January 14, 2010; Accepted March 30, 2010

DOI: 10.3892/or_00000972

\begin{abstract}
The tumor suppressor p53 is a transcription factor that induces the transcription of various target genes in response to DNA damage and it protects the cells from malignant transformation. In this study, we performed cDNA microarray analysis and found that the transmembrane protein containing sushi domain (TMPS) gene, which encodes a putative type I transmembrane protein, is a novel p53-target gene. TMPS contains a sushi domain in the extracellular region, which is associated with protein-protein interaction. TMPS expression is induced by endogenous p53 under genotoxic stress in several cancer cell lines. Reporter assay revealed p53-dependent transactivation of the p53 binding-sites (BSs) located in the intron 1 of TMPS. Chromatin immunoprecipitation (ChIP) assay showed that $\mathrm{p} 53$ binds to these BSs in vivo. Overexpression of TMPS induced apoptosis through the activation of caspase-3, 8, and 9 in various cancer cell lines. Moreover, $\gamma$-irradiation induced the expression of TMPS mRNA in the spleen and colon of $\mathrm{p} 53^{+/+}$mice but not in those of $\mathrm{p} 53^{-/-}$mice. These data indicate that TMPS may play a role in 53-dependent apoptosis under DNA damage condition.
\end{abstract}

\section{Introduction}

p53 is called as the 'Guardian of the genome' because it is stabilized in response to DNA damage or critical cellular stress, and it prevents the malignant transformation of cells by inducing the expression of various kinds of target genes. Since $p 53$ is mutated by genetic alterations such as mutations or deletions in more than half human cancers, p53 is indispensable for cancer research and has attracted the attention of many oncologists. Many target genes of p53 have been isolated; these target genes are involved in at least four main physiological functions such as cell cycle regulation, DNA

Correspondence to: Dr Hirofumi Arakawa, Cancer Medicine and Biophysics Division, National Cancer Center Research Institute, 5-1-1 Tsukiji, Chuo-ku, Tokyo 104-0045, Japan

E-mail: harakawa@ncc.go.jp

Key words: p53 target gene, TMPS, apoptosis, sushi domain repair, apoptosis, and anti-angiogenesis $(1,2)$. The p21/wafl gene is considered to be one of the most important p53 target genes because it is essential for p53-dependent cell cycle arrest at G1.p53R2, which supplies nucleotides for DNA synthesis, facilitates the repair of DNA damage. Several mitochondrial proteins including BAX, Noxa, and p53regulated apoptosis-inducing protein 1 (p53AIP1) contribute to the release of cytochrome $\mathrm{c}$ from mitochondria. Other proteins such as Fas/ApoI and unc-5 homolog B (UNC5B) are also associated with apoptosis. Brain-specific angiogenesis inhibitor 1 (BAI1) and thrombospondin 1 (TSP1) suppress angiogenesis and inhibit tumor growth. In our previous studies, we have isolated and characterized additional p53 target genes by using cDNA microarray technology, and we have reported the p53-dependent transcription of genes encoding deafness, autosomal dominant 5 (DFNA5), Semaphorin 3F (SEMA3F), B cell linker protein (BLNK), and unc-5 homolog A (UNC5A) (3-6). More studies on these target genes would clarify the physiological function of $p 53$ as a tumor suppressor gene.

Sushi domains are also known as complement control protein modules or short consensus repeats. Sushi domain contains approximately 60 amino acid residues and four cysteines (Cys); the 1st and 3rd, and the 2nd and 4th Cys residues in each repeat are linked by disulfide bonds (7-9). Proteins with these domains are included in a family which is a part of one of the largest protein superfamilies that includes proteins involved in the complement system such as complement factor $\mathrm{H}$ and complement receptor type I $(10,11)$; blood coagulation factors such as coagulation factor XIII (12); adhesion proteins such as L-selectin (13); and cytokine receptors such as IL-2 or IL-15 receptors $(14,15)$. Since the above mentioned proteins can bind to other proteins, it appears that the sushi domain often functions as a protein-binding module and endows the protein with different functions. The presence of ten sushi domain repeats in the $\beta$-subunit of the coagulation factor XIII (FXIII-B) allow for the binding of FXIII-B to the $\alpha$-subunit of coagulation factor XIII or FXIII-B homodimer assembly (12). Furthermore, sushi domain is essential for binding the IL-15 receptor (IL-15R) to its ligand IL-15 (15).

In this study, we report a novel p53 target gene, namely, transmembrane protein containing sushi domain (TMPS), which encodes a sushi domain-containing protein in the 
extracellular region and regulates apoptosis with caspase activation.

\section{Materials and methods}

Cell lines. HepG2 (hepatoblastoma), H1299 (lung cancer), LS174T, HCT116 (colon cancer), HEK293 (human kidney cell) were purchased from American Type Culture Collection (ATCC, Manassas, VA, USA), respectively. LC176 was a gift from Dr T. Takahashi (Aichi Cancer Center, Japan). All cell lines were cultured under the conditions recommended by their respective depositors.

Complementary DNA (cDNA) microarray. Total cellular RNA was extracted at the indicated time points $(0,6,12,24$ and $48 \mathrm{~h})$ from HepG2 cells after infection with adenoviral vectors at $30 \mathrm{MOI}$ (multiplicity of infection) designed to express either wild-type p53 (Ad-p53-WT), p53-46F (Ad-p53-46F), in which Ser-46 is replaced with phenylalanine (16), or enhanced green fluorescent protein (EGFP) (Ad-EGFP). For gene expression profiling, GeneChip Human Genome U133A and U133B microarrays (Affymetrix, Santa Clara, CA, USA) were used that contain 22,215 and 22,577 probe sets, respectively, to examine a total of about 39,000 transcripts. Target cRNA for microarray hybridization was prepared from $5 \mu \mathrm{g}$ of total RNA according to the manufacturer's instructions using a BioArray RNA transcript labeling kit (Enzo Diagnostics, Farmingdale, NY, USA). Hybridization to the microarrays, washing and staining with the antibody amplification procedure, and scanning was also carried out according to the manufacturer's instructions. The expression value of each gene was calculated and normalized using Affymetrix Microarray Suite software version $5.0(3)$.

RNA interference. We established the p53-knock down (p53$\mathrm{KD})$ and the control (Cont) cell lines as described previously (3-6). Briefly, HepG2, LS174T, HCT116 and LC176 cells were infected with SI-MSCV-puro-H1R-p53Ri retrovirus for down-regulation of p53 expression and with SI-MSCV-puroH1R-control retrovirus for negative control. Each stable cell line was established after selection with $1 \mu \mathrm{g} / \mathrm{ml}$ puromycin.

Plasmids. The entire coding sequence of TMPS cDNA was amplified by PCR using KOD-plus DNA polymerase (TOYOBO, Osaka, Japan) and inserted into pCR-Blunt IITOPO vector (Invitrogen, Carlsbad, CA, USA). After confirmation of sequencing, the EcoRI-digested DNA fragment was cloned into pcDNA 3.1(+) (pcDNA-TMPS sense/ antisense) (Invitrogen) and pCMV-Tag2B (Tag2B-TMPS) (Stratagene, La Jolla, CA, USA).

Semi-quantitative reverse transcription-polymerase chain reaction $(R T-P C R) . \mathrm{p} 53-\mathrm{KD}$ and Cont cell lines were treated with adriamycin $(1 \mu \mathrm{g} / \mathrm{ml}, 2 \mathrm{~h})$. Total RNAs were isolated at the indicated time $(0,6,12,24$ and $48 \mathrm{~h})$ using TRIzol reagent (Invitrogen). Furthermore total RNAs were also extracted from irradiated mouse tissues. A $5 \mu \mathrm{g}$ aliquot of total RNA was reverse-transcribed and the PCR was run in the exponential region (18-28 cycles) to allow semi-quantitative comparisons among cDNAs developed from identical reactions. Oligonucleotide primer sequences were as follows: Human-TMPS- forward; 5'-GCTCAGGGAACCGCCAACT-3', HumanTMPS-reverse; 5'-AGGCAGATGGCGAGAATCT-3'. MouseTMPS-forward; 5'-GCAGGCAGTGTCATCGAGTA-3', Mouse-TMPS-reverse; 5'-TTGGCTGCAGCAGTACAAAC-3'.

Western blot analysis. HEK293 cells were transfected with Tag2B-TMPS. Twenty-four hours later, the cells were collected and lysed in chilled Radio-immuno-protein-assay (RIPA) buffer [1\% Nonidet-P40 (NP-40), 50 mM Tris-HCl pH 8.0, $150 \mathrm{mM} \mathrm{NaCl}, 0.5 \%$ Deoxycholate, $0.1 \%$ Sodium dodecyl sulfate (SDS), protease inhibitor cocktail]. Homogenates were subjected to SDS-polyacrylamide gel electrophoresis and blotted onto Hybond-P (GE Healthcare Bio-Sciences, Piscataway, NJ, USA). The blots were incubated with anti-Flag M2 antibody (Sigma, St. Louis, MO, USA) or anti-ß-actin (Sigma), then visualized by ECL Western blotting detection reagents (GE Healthcare).

Gene reporter assay. The plasmid constructs containing the putative p53 BSs of TMPS were termed pGL-BS1, BS2, or BS3, respectively. Each genomic fragment containing putative p53BS was amplified by PCR and cloned into pGL3-promoter vector (Promega, Madison, WI, USA). H1299 cells were plated in 6 -well culture dishes $\left(1 \times 10^{5}\right.$ cells/dish), then each pGL-BS vector containing $\mathrm{BS} 1, \mathrm{BS} 2$ or $\mathrm{BS} 3$ was co-transfected with either wild-type p53, mutant p53 (R175H) or empty expression vector (mock) in combination with pRL-CMV vector (Promega). Twenty-four hours after transfection, cells were lysed and subjected to the Dual Luciferase assay system (Promega). Quantification of luciferase activities was carried out manually with a luminometer.

Chromatin immunoprecipitation (ChIP) assay. ChIP assay was performed using the ChIP assay kit (Upstate, Lake Placid, NY, USA) as recommended by the manufacture. H1299 cells $\left(1 \times 10^{6}\right.$ cells) were infected with Ad-p53-WT and Ad-EGFP at $30 \mathrm{MOI}$. After $24 \mathrm{~h}$, genomic DNA and protein were crosslinked by adding formaldehyde ( $1 \%$ final concentration) directly into the culture medium and incubated for $15 \mathrm{~min}$ at $37^{\circ} \mathrm{C}$. Cells were lysed in $200 \mu \mathrm{l}$ SDS lysis buffer with a protease inhibitor cocktail and sonicated to generate DNA fragments 200-1,000 bp long. After centrifugation, the cleared supernatant was diluted with ChIP assay buffer and incubated at $4^{\circ} \mathrm{C}$ with the specific antibody with rotation. Immune complexes were precipitated, washed, and eluted as recommended. DNA-protein cross links were reversed by heating to $65^{\circ} \mathrm{C}$ for $5 \mathrm{~h}$. DNA was phenol-extracted, ethanol-precipitated and resuspended in $50 \mu 1$ of water. Two microliters of each sample were subjected to PCR amplification with 30 cycles. PCR was done using the following specific primers containing p53 BSs, 5'-ACCCTGAAGGTCTGGGAGA-3' (BS1, forward), 5'-CCTATGCAGATCTGTCCATC-3' (BS1, reverse), 5'-CATAGGAGTTACGCACACAC-3' (BS2, forward), 5'-GCACAAAGGTTGGTACTCAG-3' (BS2, reverse), 5'-CTGCTGTTAATGGACCATACT-3' (BS3, forward), 5'-GGGCTGTGGTTCTTATACCT-3' (BS3, reverse).

Recombinant adenovirus. The adenovirus expressing TMPS was prepared using adenovirus expression vector kit (Takara, 
A

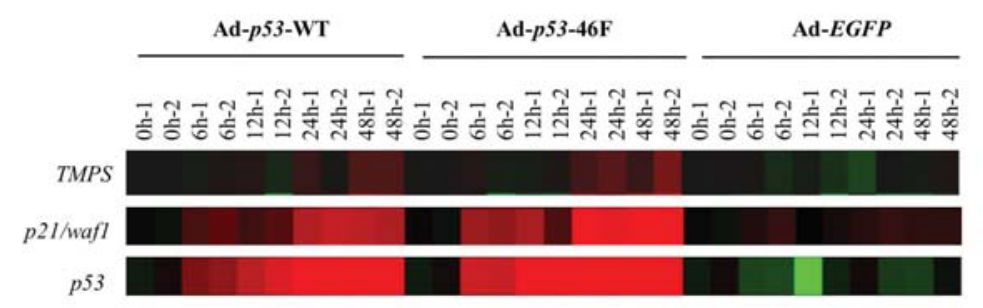

B

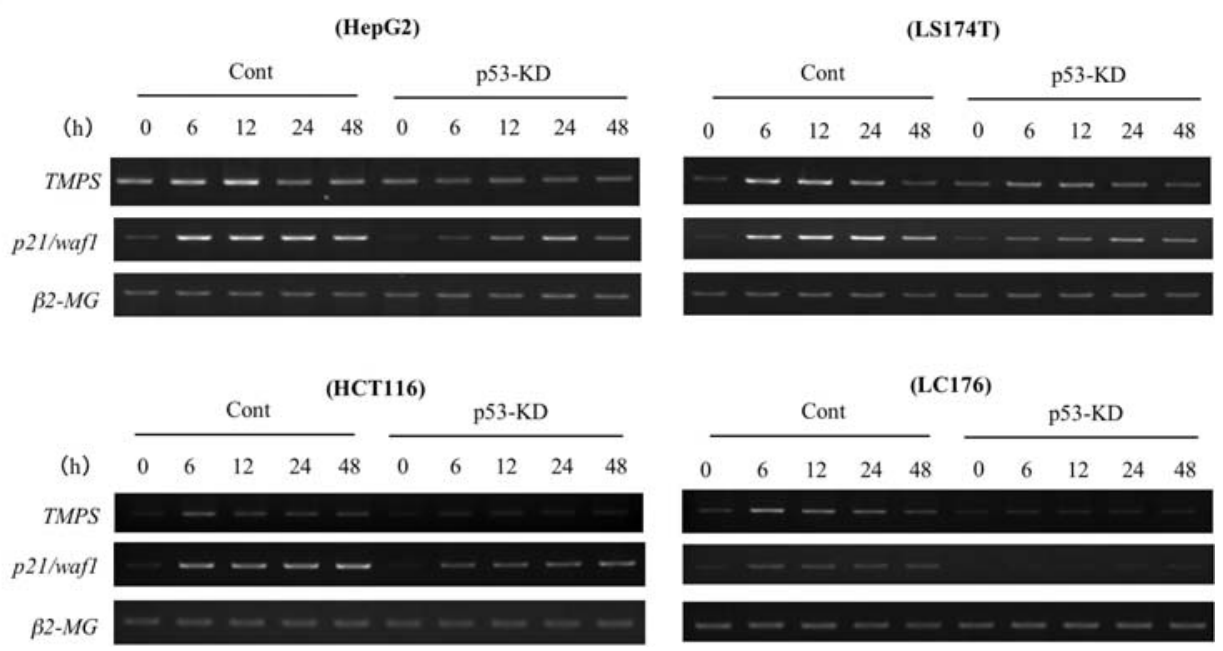

Figure 1. Identification of TMPS as a p53-inducible gene. (A) Microarray analysis of TMPS expression. The green and red signals on the microarray indicate the intensity of expression of TMPS, p21/waf1, and $p 53$ mRNA in HepG2 cells at the indicated times after infection with Ad-p53-WT, Ad-p53-46F, and Ad-EGFP. Red signal indicates upregulation and green signal indicates downregulated expression of genes. (B) Induction of TMPS by endogenous p53. Adriamycintreated p53-KD and Cont cells (HepG2, LS174T, HCT116, and LC176 cells) were subjected to RT-PCR.p21/waf1 and $\beta 2-M G$ were used as positive control and quantity control, respectively.

Otsu, Japan) according to the manufacturer's instructions. In brief, blunt-ended full length TMPS were also cloned into the Smil site of the cosmid pAxCAwtit, which contains the CAG promoter and the entire genome of type 5 adenovirus except for E1 and E3 regions, then transfected to HEK293. Viruses were propagated in the 293 cells and purified as described previously (16). Expression level of TMPS mRNA was evaluated by RT-PCR at $24 \mathrm{~h}$ post infection to H1299 cells at the indicated MOIs.

Apoptosis assay. Various cancer cell lines were infected with Ad-TMPS at indicated MOIs (0, 30, 60 and $100 \mathrm{MOI})$. After $72 \mathrm{~h}$ of infection, the cells were collected and fixed with $70 \%$ ethanol. Fixed samples were centrifuged, treated with RNase $(0.2 \mathrm{mg} / \mathrm{ml})$, and resuspended in propidium iodide $(50 \mu \mathrm{g} / \mathrm{ml})$. The stained cells were analyzed on a FACScan flow cytometer (Beckton-Dickinson, Franklin Lakes, NJ, USA). The subG1 fraction of the cells was counted as the apoptotic cells, and the proportion of the apoptotic cells to the total cells was indicated as percentage.

Caspase activity. The labeled synthetic substrates $\mathrm{N}$-acetylAsp-Glu-Val-Asp p-nitroanilide (Ac-DEVD-pNA) for caspase 3, N-acetyl-Ile-Glu-Pro-Asp p-nitroanilide (Ac-IEPDpNA) for caspase 8 and N-acetyl-Leu-Glu-His-Asp-7-amido4-fluoromethylcoumarin (Ac-LEHD-AFC) for caspase 9 were purchased from Sigma. Cell lysates were prepared from
LS174T cells 36 or $60 \mathrm{~h}$ after infection with Ad-TMPS, Ad$E G F P$ or Ad-p53, and reacted with each substrate in the assay buffer [50 mM 4-(2-hydroxyethyl)-1-piperazineethanesulfonic acid (HEPES), pH 7.4, $100 \mathrm{mM} \mathrm{NaCl}, 0.1 \% 3-$ [(3-Cholamidopropyl) dimethylammonio] propanesulfonate (CHAPS), $1 \mathrm{mM}$ ethylenediaminetetraacetic acid (EDTA), $10 \%$ glycerol, $5 \mathrm{mM}$ dithiothreitol] at $37^{\circ} \mathrm{C}$. The free pNA cleaved from the substrate was detected as the caspase activity by monitoring the optical density at $405 \mathrm{~nm}$. In this system, caspase activity was presented as the ratio compared with the control from the cells infected with Ad-EGFP.

Immunocytochemistry. Tag2B-TMPS was transfected into H1299 cells in an 8-well chamber slide. Twenty-four hours later, the cells were fixed with methanol at $-20^{\circ} \mathrm{C}$ for $15 \mathrm{~min}$, then blocked with $3 \%$ bovine serum albumin (BSA) in phosphate buffered saline (PBS) for $1 \mathrm{~h}$ at room temperature (RT). Anti-Flag M2 antibody (1:250) was applied for $1 \mathrm{~h}$ at RT, followed by goat anti-mouse fluorescein isothiocyanate (FITC) (1:500, Vector Laboratories, Burlingame, CA, USA) and mounted with propidium iodine (PI), then visualized using Radiance 2000 confocal microscope (Bio-Rad Laboratories, Hercules, CA, USA).

p53-knockout mice. p53-deficient mice were a gift from Dr S. Aizawa, Center for Developmental Biology, RIKEN, Japan (17). $p 53^{+/+}$and $p 53^{-/-}$mice $(8-12 \mathrm{w})$ were irradiated by 
A

\begin{tabular}{|l|l|l|l|l|l|l|l|}
\hline aa & \multicolumn{1}{|c|}{40} & 42 & \multicolumn{1}{|c|}{102} & 120 & 142 & 303 \\
$\mathrm{NH} 2$ & Sp & & Sushi & & TM & \\
\hline
\end{tabular}

B

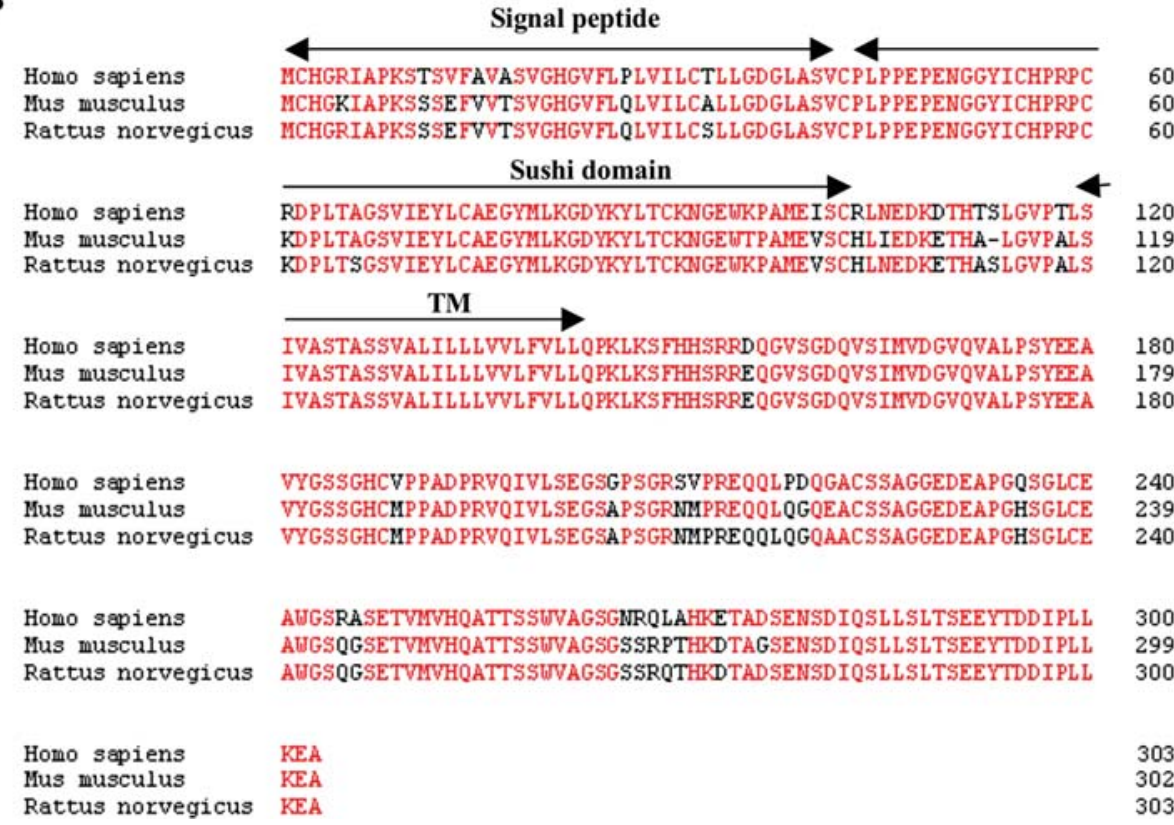

Figure 2. (A) Structure of TMPS. TMPS contains 303 amino acids. The structure of TMPS includes a few motifs or domains: Sp, signal peptide motif; Sushi, sushi domain and TM, transmembrane domain. Each number indicates the amino acid number. (B) Comparison between TMPS amino acid sequences of humans and other organisms. Alignment of human, mouse, and rat homologs of TMPS is shown. Consensus sequences are indicated in red. The arrows indicate either signal peptide motif, sushi domain, or transmembrane domain (TM), respectively.

$10 \mathrm{~Gy}$. Then the mice were euthanized and harvested for RNA extraction at indicated times $(0,4,24$ and $48 \mathrm{~h})$ after irradiation. Total RNAs purified from the homogenized tissues (spleen, colon, thymus and brain) were reverse-transcribed and subjected to RT-PCR analysis as described above. All mouse procedures were carried out according to the recommendations of the Institutional Animal Care and Use Committee of the National Cancer Center at Tsukiji, Japan.

\section{Results}

Identification of TMPS as a novel p53-inducible gene. To identify additional transcriptional targets of p53 in the human genome, we used cDNA microarrays and screened p53inducible transcripts according to previously described methods $(3,5)$. In these experiments, $p 21 /$ wafl, whose expression was clearly induced by $\mathrm{p} 53$, was used as the positive control; the results indicated that $\mathrm{p} 53$ upregulated the expression of approximately 100 genes. We selected one of these genes, namely, TMPS, whose expression level was especially elevated by either adenovirus vector expressing wild-type p53 (Adp53-WT) or adenovirus vector expressing mutant p53 (Adp53-46F), in comparison with adenovirus vector expressing green fluorescent protein (Ad-EGFP) in a time-dependent manner (Fig. 1A). p53-46F was previously demonstrated to be an active form of p53 with an enhanced ability to induce apoptosis (16). The microarray results were confirmed by
RT-PCR. We evaluated the induction of TMPS mRNA by endogenous p53. p53-knock down (p53-KD) and control (Cont) cell lines of HepG2, A549, HCT116, and LC176 were treated with adriamycin, and then the cells were harvested for total RNA isolation. Adriamycin treatment induced the expression of TMPS mRNA, which was stronger in Cont cells than in p53-KD cells (Fig. 1B); hence, the expression of TMPS mRNA was induced in response to DNA damage in a p53dependent manner.

Structure of TMPS. The TMPS gene is located at chromosome 14q24.1; its sequence has been deposited in the National Center for Biotechnology Information (NCBI) under the accession number NM_014734 with unknown function. Basic Local Alignment Search Tool (BLAST) analysis revealed that TMPS contains a signal peptide (Sp) motif at the N-terminus; a sushi domain (42-102 aa), which functions as a proteinbinding module; and a transmembrane domain (120-142 aa) (Fig. 2A). The identity values of TMPS amino acid sequence reveal that the protein sequence is well conserved between human and rodents (human vs. mouse, $89 \%$ identity; human vs. rat, $90 \%$ identity) (Fig. 2B).

TMPS as a direct transcriptional target of p53. We identified three putative p53 binding sites in the genomic region of TMPS. One binding site (BS1) was in the promoter region and the remaining two (BS2 and BS3) were in intron 1 
A

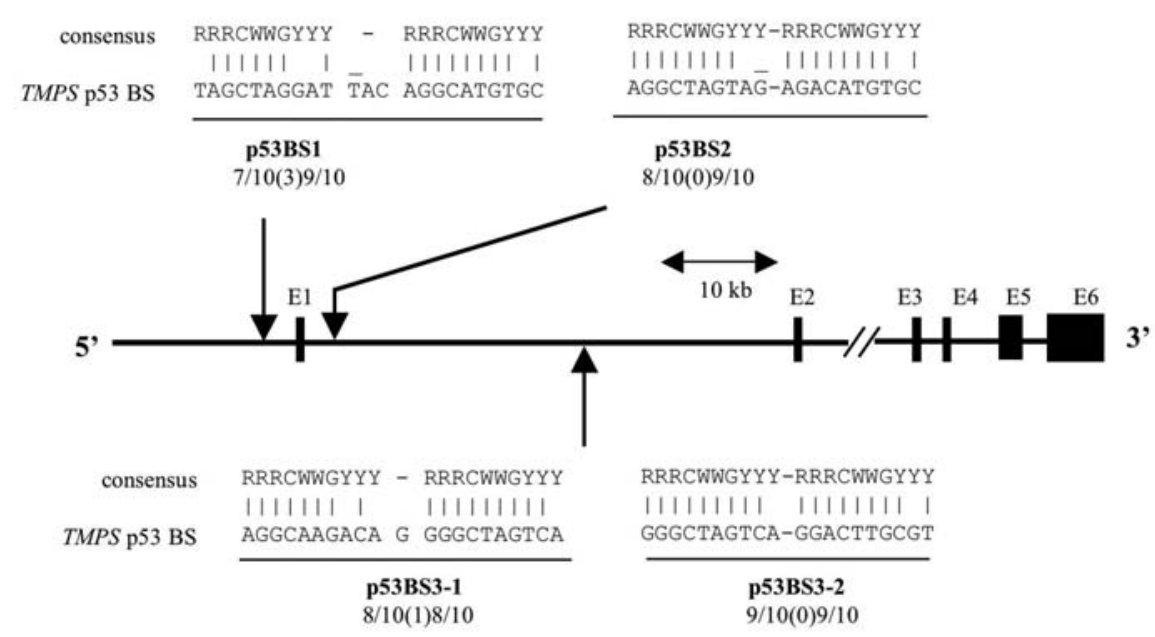

B

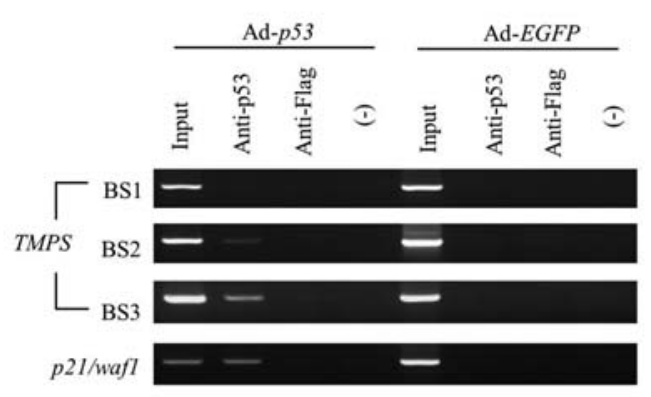

C

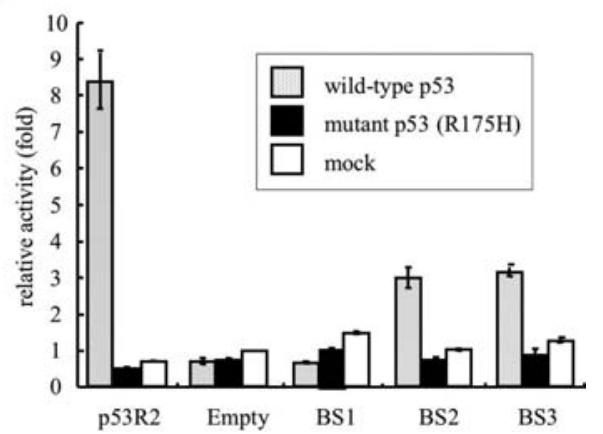

Figure 3. TMPS is the direct target of p53. (A) Genomic structure of TMPS is indicated. The three potential p53-binding sites (BS1, BS2 and BS3) are shown in comparison with the consensus p53-binding sequence. R, purine; W, A or T; Y, pyrimidine. E indicates exon. (B) ChIP assay. Ad-p53-infected (lanes 1-4) or Ad-EGFP-infected (lanes 5-8) H1299 cells were isolated and a cell lysate was prepared. A protein complex of p53 and genomic DNA was precipitated using anti-p53 antibody (lanes 2 and 6), anti-Flag antibody (lanes 3 and 7), or in the absence of an antibody (lanes 4 and 8) as negative controls. The input chromatins, which are portions of the sonicated chromatin before immunoprecipitation, are shown in lanes 1 and 5.p21/waf1-BS was used as positive control. (C) Reporter assay. Reporter plasmids containing BS1, BS2, or BS3 were co-transfected with plasmids expressing either wild-type p53, mutant p53 (R175H), or empty vector (mock). pGL-p53R2 was used as positive control and pGL-empty as negative control. The relative luciferase activities of wild-type p53 and mutant p53 were compared with that of empty pGL vector.

(Fig. 3A). To evaluate the in vivo binding of p53 with p53BS, we performed a chromatin immunoprecipitation (ChIP) assay. The DNA fragments precipitated from Ad-p53infected H1299 cells by using the anti-p53 antibody revealed that the fragments contained both BS2 and BS3 but not BS1, thereby indicating that $\mathrm{BS} 2$ and $\mathrm{BS} 3$ interact with $\mathrm{p} 53$ in vivo (Fig. 3B). We performed a reporter assay to determine whether these sequences actually exhibit p53-dependent transcriptional activity. The results showed that luciferase activity was enhanced by co-transfection of pGL-BS2 or BS3 vector with the wild-type p53 expression vector but not with the mutant p53 vector, thereby indicating that BS2 and BS3 exhibit p53dependent transcriptional activity (Fig. 3C). These results confirmed that TMPS is a direct target of p53.

TMPS plays a role in the suppression of cell growth. To investigate the potential effect of TMPS on cell death and proliferation, we performed a colony formation assay. Before the assay, we determined the expression level of sense/ antisense TMPS by using plasmids designed to express these genes. We confirmed the expression of the plasmid vectors that express sense-TMPS or antisense-TMPS (Fig. 4A). The number of G418-resistant colonies was compared among the
sense-TMPS-, antisense-TMPS-, and empty-transfected cells at 2 weeks after transfection. As shown in Fig. 4B and C, the colony number of sense-TMPS-transfected cells was significantly reduced as compared to that of antisense-TMPS- or empty-transfected cells. Therefore, we suspected that TMPS may possess apoptotic activity.

TMPS induces apoptosis. To evaluate the role of TMPS in apoptosis, we generated adenoviral vector designed to express TMPS (Ad-TMPS). We confirmed that this vector allows good expression of TMPS mRNA in a dose-dependent manner (Fig. 5A). Then, several cancer cell lines cells (HCT116, HepG2, LS174T, H1299, and U373MG) were infected with Ad-TMPS at various MOIs, and apoptotic cells were analyzed by fluorescence-activated cell sorting (FACS) at the indicated times. Regardless of the p53 status, transfection with AdTMPS increased apoptosis in all cell lines in a MOI-dependent manner (Fig. 5B). Next, we measured the caspase activity (caspase 3, 8 and 9) using LS174T cells. The 36-h activity of each caspase was higher in Ad-TMPS-infected cells than that in Ad-EGFP-infected cells (100 MOI) or Ad-p53-infected cells (10 MOI) (Fig. 5C). However, the caspase activities declined $60 \mathrm{~h}$ after infection because most Ad-TMPS-infected 
A

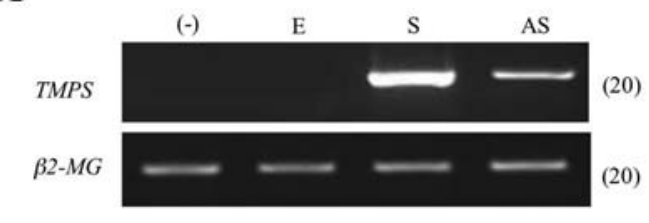

B

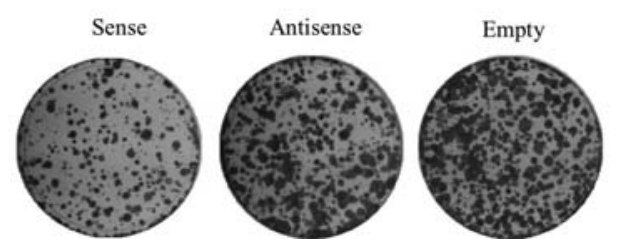

C

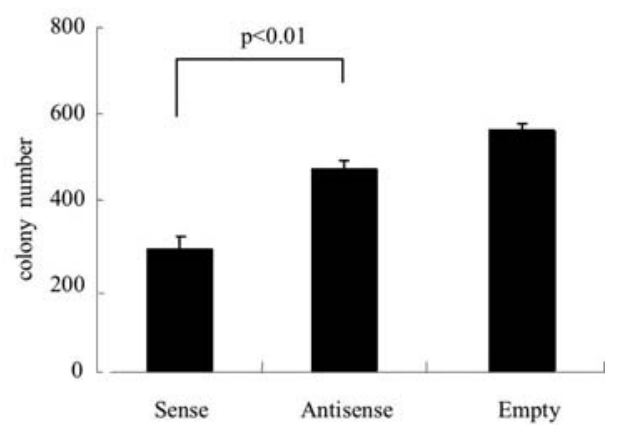

Figure 4. Colony formation assay. (A) The expression of TMPS in H1299 cells was analyzed by RT-PCR. The results indicate the expression levels of TMPS mRNA in H1299 cells $24 \mathrm{~h}$ after transfection with expression vectors. (-) indicates no transfection, (+), transfection with empty (E)/pcDNA3.1; S, transfection with pcDNA-TMPS-sense; and AS, transfection with pcDNA-TMPS-antisense. The numbers in parenthesis indicate the number of PCR cycles. (B) Representative G418-resistant colonies of H1299 cells transfected with each plasmid are shown. (C) The average number of colonies in triplicate samples is indicated with error bars indicating one standard deviation (SD) on histograms.

A

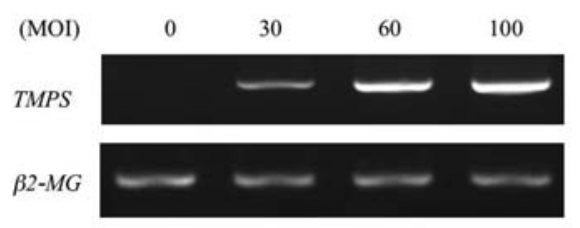
(15) (20)

B

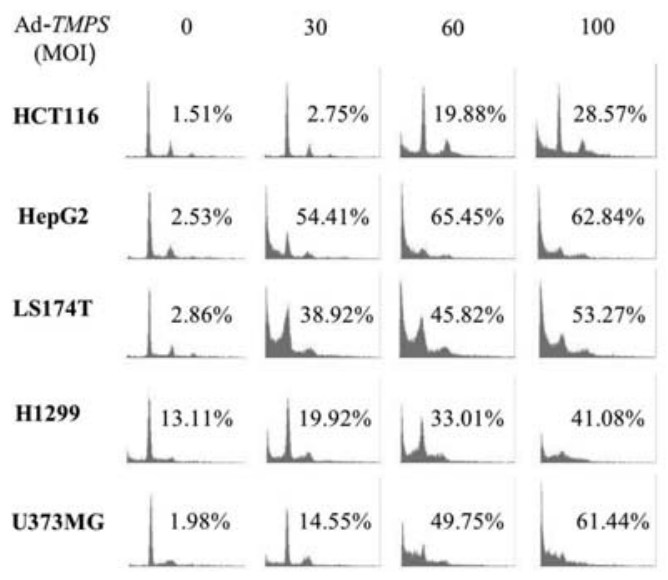

C
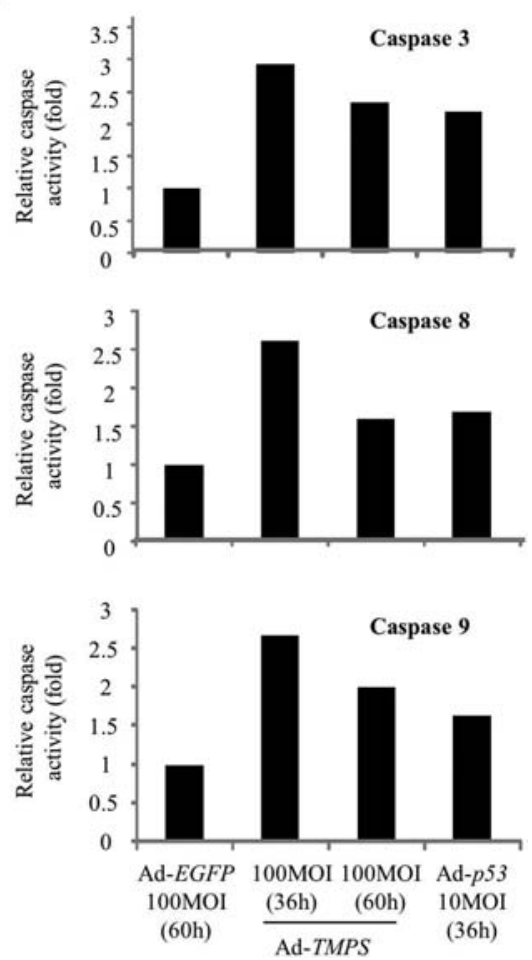

Figure 5. Apoptosis by TMPS. (A) The expression level of TMPS mRNA in H1299 cells at 24 h after infection with Ad-TMPS. The numbers in parenthesis indicate the number of PCR cycles. (B) Apoptotic cell death caused by Ad-TMPS. HCT116, HepG2, LS174T, H1299, and U373MG cells were infected with Ad-TMPS (0, 30, 60 and $100 \mathrm{MOI})$ and analyzed by FACS scan $72 \mathrm{~h}$ after infection. DNA histogram for each cell type is shown and the percentage of the respective subG1 fraction is also shown. (C) Caspase activity. LS174T cells were infected with either Ad-TMPS, Ad-p53, or Ad-EGFP at the indicated MOIs. The cells were collected and the activities of caspase 3,8 , and 9 were determined after 36 or $72 \mathrm{~h}$ after infection. The relative ratio of each sample was calculated in comparison with the caspase activity of Ad-EGFP-infected cells.

cells were already killed by then. These results suggest that TMPS caused apoptotic cell death with caspase activation.

Localization of TMPS. BLAST analysis suggested that TMPS is a membrane protein. To determine the localization of TMPS, we performed immunofluorescent analysis. We performed
Western blotting to confirm the expression of TMPS in HEK 293 cells transfected with Tag2B-TMPS (Fig. 6A). Then, we transfected this plasmid into H1299 cells and subjected the cells to immunofluorescent analysis. As predicted, fluorescent signals were detected on the surface of the cells, indicating that TMPS was a membrane protein (Fig. 6B). 
A

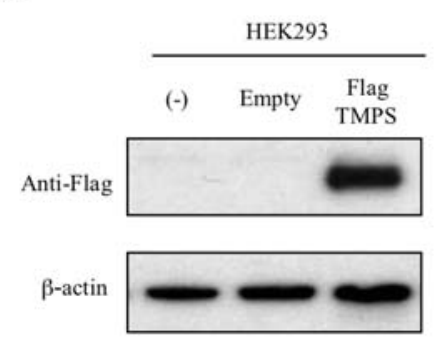

B

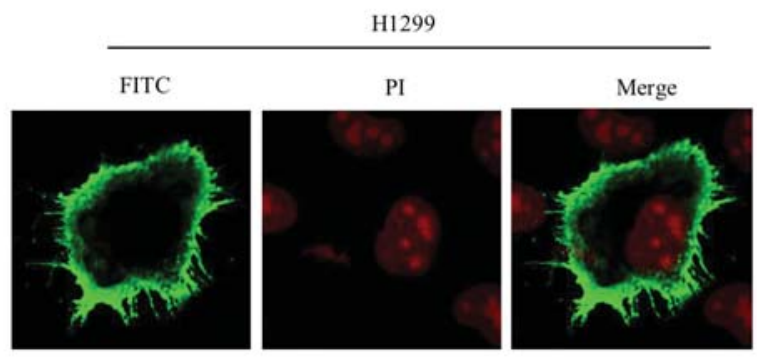

C

(Brain)

(Spleen)
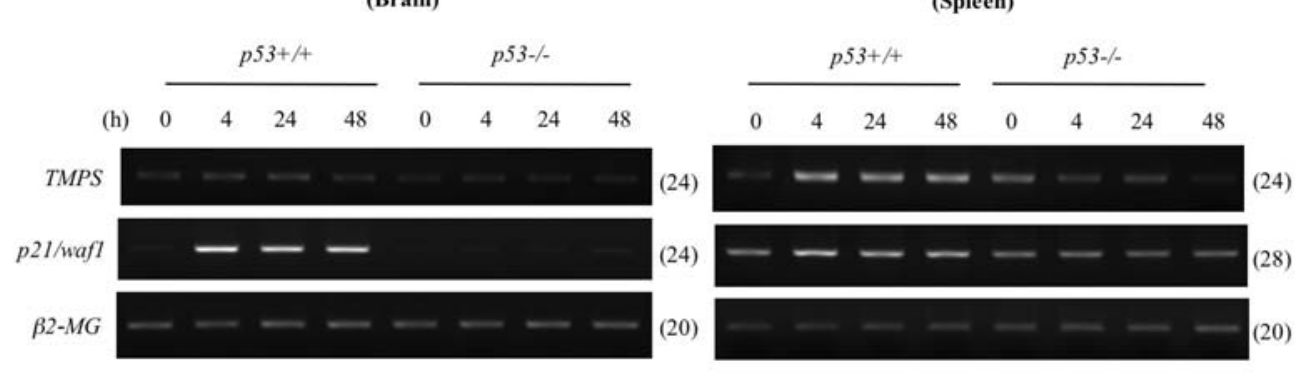

(Colon)

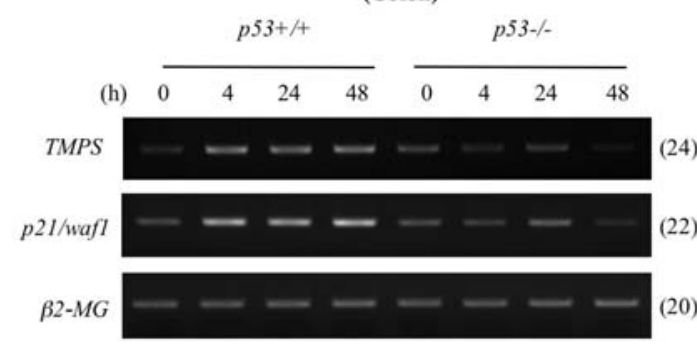

(Thymus)

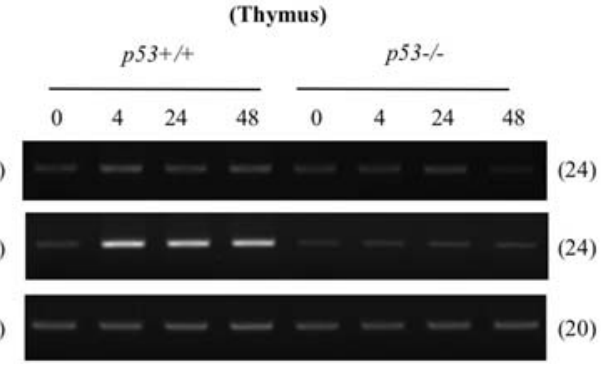

Figure 6. Localization and potential in vivo role of TMPS. (A) Expression of Flag-tagged TMPS was confirmed by Western blot analysis. 3 -actin was used as the loading control. (B) Tag2B-TMPS expressing Flag-tagged TMPS was transfected into H1299 cells. The cells were stained with anti-Flag antibody (FITC), counterstained by propidium iodine (PI), and visualized by confocal microscopy. (C) Irradiated $p 53^{+/+}$or $p 53^{-/-}$mice were euthanized at the indicated time points. cDNAs from various tissues including the spleen, colon, thymus and brain were analyzed by RT-PCR. $p 21 / w a f 1$ was used as positive control and $\beta 2-M G$ as quality control. The numbers of RT-PCR cycles for each sample are indicated in parentheses.

TMPS functions as an in vivo mediator of p53 response after DNA damage. To assess whether TMPS truly functions as an in vivo mediator of p53 action, we determined the p53dependent inducibility of TMPS by using $p 53$-knockout mice. We irradiated $p 53^{+/+}$and $p 53^{-/-}$mice with 10 Gy of $\gamma$-rays, and isolated total RNA from the spleen, colon, brain, and thymus of the irradiated mice at the indicated times. Interestingly, we observed a strong p53-dependent induction of TMPS expression in the spleen and colon. Further, the expression of TMPS mRNA in the brain and thymus was also slightly increased. These results suggest that TMPS plays an important role in DNA damage-induced p53-dependent apoptosis in vivo (Fig. 6C).

\section{Discussion}

p53 mainly prevents the development of cancer by inducing apoptosis. In response to DNA damage, p53 suppresses cell growth through the cell cycle and allows DNA repair. However, in the event of severe damage, p53 induces apoptosis and eliminates seriously damaged cells. Among the various p53 target genes isolated so far, several genes appear to be apoptosis-related genes. These genes are classified into at least 3 groups as follows: genes in the mitochondrial pathway (Bax, Puma, Noxa, p53AIP1, PIG3, POX ALDH4), death receptor pathway (Killer/DR5,FAS), and dependence receptor pathway (UNC5B termed p53RDL1, UNC5A) (2). Several additional target genes that are not directly involved in these pathways also mediate p53-dependent apoptosis. Several specific domains in the proteins encoded by these genes are known to play a critical role in apoptosis. For example, the $\mathrm{BH} 3$ domain in Bcl-2 family proteins, including Bax and Puma, triggers a mitochondrial apoptotic event and affects the release of cytochrome c from mitochondria (18). Killer/DR5 and FAS receptor, which contain the death domain, bind to their ligands and induce cell death with the recruitment of an adaptor molecule and procaspase 8 (19). The death domain in dependence receptors such as UNC5A or UNC5B is also involved in caspase-activated apoptosis in the absence of ligand netrin-1 $(6,20)$. In this study, we report that TMPS, which is encoded by a novel p53 target gene, contains a sushi domain in its extracellular region and is involved in apoptosis with caspase-activation. This is the first report of a sushi domain-containing p53-target that plays a role in apoptosis.

It was believed that sushi domain functions as a proteinbinding module and is involved in receptor-ligand interactions 
(7-9). Interestingly, emerging evidence suggests that sushi domain may also be involved in apoptosis. Sushi domain in the extracellular region of IL15-R $\alpha$ is strongly associated with cell survival and apoptosis (15). Amino acid substitution of the 1 st or 4th cysteine in the sushi domain abolished the IL-15-binding activity of IL15-R $\alpha$, and resulted in the loss of IL-15-induced proinflammatory cytokine production and anti-apoptotic activity (15). Further, the gene encoding tumor suppressor drs, which contains three sushi domain repeats, induces apoptosis in several human cancer cells with caspase activation (21). The deletion of these repeats apparently decreased apoptosis, indicating that sushi domains may be the key functional domains in apoptosis; however, the binding partners of sushi domain are unknown. Drs-induced apoptosis involved sequential activation of caspases 12, 9, and 3; however, neither cytochrome-c release nor activation of caspase 8 was observed, suggesting that mitochondrial pathway does not mediate drs-induced apoptosis (22). In contrast, in the present study, TMPS activated not only caspase 9 and 3 but also caspase 8, indicating that TMPSinduced apoptosis may involve the participation of various kinds of apoptotic pathways. Since TMPS localizes in cytoplasmic membrane, caspase 8 could also be activated by TMPS, similarly to death receptor pathway. These results indicate that TMPS is one of the apoptotic regulators induced by $\mathrm{p} 53$.

Indeed, apoptosis is one of the core functions regulated by p53, and it is very important to clarify its mechanism, including the role of TMPS. Further, detailed investigation is necessary to reveal the role of this interesting molecule in tumorigenesis.

\section{Acknowledgements}

We thank H. Ichikawa, T. Ohta, and M. Ohki for the microarray analysis. We also thank S. Usuda and I. Hyo for their excellent technical assistance. H.A. is supported in part by grants from the Ministry of Health, Labor and Welfare, Japan, and the Ministry of Education, Culture, Sports, Science and Technology, Japan.

\section{References}

1. Nakamura Y: Isolation of p53-target genes and their functional analysis. Cancer Sci 95: 7-11, 2004.

2. Arakawa H: p53, apoptosis and axon-guidance molecules. Cell Death Differ 12: 1057-1065, 2005.

3. Masuda Y, Futamura M, Kamino H, Nakamura Y, Kitamura N, Ohnishi S, MiyamotoY, Ichikawa H, Ohta T, Ohki M, Kiyono T, Egami H, Baba H and Arakawa H: The potential role of DFNA5, a hearing impairment gene, in p53-mediated cellular response to DNA damage. J Hum Genet 51: 652-664, 2006.
4. Futamura M, Kamino H, Miyamoto Y, Kitamura N, Nakamura Y, Ohnishi S, Masuda Y and Arakawa H: Possible role of semaphorin $3 \mathrm{~F}$, a candidate tumor suppressor gene at $3 \mathrm{p} 21.3$, in p53regulated tumor angiogenesis suppression. Cancer Res 67: 1451-1460, 2007.

5. Kamino H, Futamura M, Nakamura Y, Kitamura N, Kabu K and Arakawa H: B-cell linker protein prevents aneuploidy by inhibiting cytokinesis. Cancer Sci 99: 2444-2454, 2008.

6. Miyamoto Y, Futamura M, Kitamura N, Nakamura Y, Baba H and Arakawa H: Identification of UNC5A as a novel transcriptional target of tumor suppressor $\mathrm{p} 53$ and a regulator of apoptosis. Int J Oncol 36: 1253-1260, 2010.

7. Lozier J, Takahashi $\mathrm{N}$ and Putnam FW: Complete amino acid sequence of human plasma B2-glycoprotein I. Proc Natl Acad Sci USA 81: 3640-3644, 1984.

8. Ichinose A, Bottenus RE, Earl W and Davie EW: Structure of transglutaminases. J Biol Chem 265: 13411-13414, 1990.

9. Kato $\mathrm{H}$ and Enjyoji $\mathrm{K}$ : Amino acid sequence and location of the disulfide bonds in bovine beta 2 glycoprotein I: the presence of five Sushi domains. Biochemistry 30: 11687-11694, 1991.

10. Ripoche J, Day AJ, Harris TJR and Sim RB: The complete amino acid sequence of human complement factor $\mathrm{H}$. Biochem J 249: 593-602, 1988.

11. O'Leary JM, Bromek K, Black GM, et al: Backbone dynamics of complement control protein (CCP) modules reveals mobility in binding surfaces. Protein Sci 13: 1238-1250, 2004.

12. Souri M, Kaetsu H and Ichinose A: Sushi domains in the B subunit of factor XIII responsible for oligomer assembly. Biochemistry 47: 8656-8664, 2008.

13. Nishimura Y, Shimojima M, Tohya Y and Miyazawa T: Molecular cloning of a cDNA encoding the feline CD62L. J Vet Med Sci 69: 81-84, 2007.

14. Rickert M, Wang X, Boulanger MJ, Goriatcheva N and Garcia KC: The structure of interleukin-2 complexed with its alpha receptor. Science 308: 1477-1480, 2005.

15. Wei X, Orchardson M, Gracie JA, et al: The sushi domain of soluble IL-15 receptor $\alpha$ is essential for binding IL-15 and inhibiting inflammatory and allogenic responses in vitro and in vivo. J Immunol 167: 277-282, 2001.

16. Nakamura Y, Futamura M, Kamino H, Yoshida K, Nakamura Y and Arakawa $\mathrm{H}$ : Identification of p53-46F as a super p53 with an enhanced ability to induce p53-dependent apoptosis. Cancer Sci 97: 633-641, 2006.

17. Tsukada T, Tomooka Y, Takai S, et al: Enhanced proliferative potential in culture of cells from p53-deficient mice. Oncogene 8: 3313-3322, 1993.

18. Chittenden T: BH3 domains: intracellular death-ligands critical for initiating apoptosis. Cancer Cell 2: 165-166, 2002.

19. Jeong EJ, Bang S, Lee TH, et al: The solution structure of FADD death domain. Structural basis of death domain interacts of Fas and FADD. J Biol Chem 274: 16337-16342, 1999.

20. Tanikawa C, Matsuda K, Fukuda S, Nakamura Y and Arakawa H: p53RDL1 regulates p53-dependent apoptosis. Nat Cell Biol 5: 216-223, 2003.

21. Yamashita A, Hakura A and Inoue H: Suppression of anchorageindependent growth of human cancer cell lines by the drs gene. Oncogene 18: 4777-4787, 1999.

22. Tambe Y, Isono T, Haraguchi S,Yoshioka-Yamashita A, Yutsudo M and Inoue H: A novel apoptotic pathway induced by the drs tumor suppressor gene. Oncogene 23: 2977-2987, 2004. 